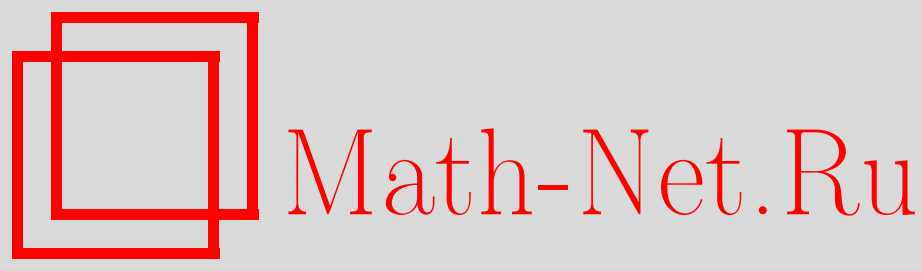

Е. В. Выборный, Туннельное расщепление спектра и билокализация собственных функций в несимметричной двойной яме, ТМФ, 2014, том 178, номер 1, 107-130

DOI: https://doi.org/10.4213/tmf8531

Использование Общероссийского математического портала Math-Net.Ru подразумевает, что вы прочитали и согласны с пользовательским соглашением http://www . mathnet.ru/rus/agreement

Параметры загрузки:

IP: 3.82 .47 .9

26 апреля 2023 г., 09:59:58

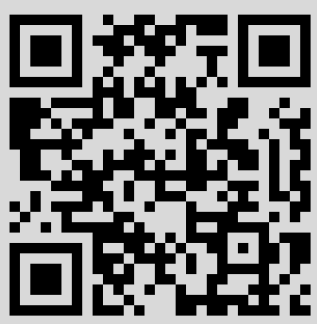




\title{
ФИЗИКА
}

Том 178, № 1

январь, 2014

(C) 2014 г.

Е. В. Выборный*

\section{ТУННЕЛЬНОЕ РАСЩЕПЛЕНИЕ СПЕКТРА И БИЛОКАЛИЗАЦИЯ СОБСТВЕННЫХ ФУНКЦИЙ В НЕСИММЕТРИЧНОЙ ДВОЙНОЙ ЯМЕ}

\begin{abstract}
Рассматривается одномерное стационарное уравнение Шредингера с гладким потенциалом, имеющим вид двойной ямы. Получен критерий наличия двойной локализации волновых функций, экспоненциального расщепления энергетических уровней и туннельной транспортации частицы в несимметричном потенциале. Получены асимптотические формулы для величины расщепления энергий, обобщающие известные формулы для случая зеркально-симметричного потенциала. Рассмотрен случай высоких энергетических уровней и случай энергий, близких к минимумам потенциала. Приведен пример туннельной транспортации в несимметричной двойной яме. Кроме того, рассмотрена задача туннельного возмущения дискретного спектра оператора Шредингера с потенциалом с одной ямой. Экспоненциально малое возмущение энергий имеет место при локальных деформациях потенциала, сосредоточенных только в классически запрещенной области. Вычислен главный член асимптотики туннельного возмущения спектра.
\end{abstract}

Ключевые слова: туннелирование, квазипересечение энергетических уровней, одномерное уравнение Шредингера, квазиклассическое приближение.

DOI: $10.4213 / \operatorname{tmf} 8531$

\section{1. ВВЕДЕНИЕ}

Квантовое туннелирование представляет интерес для многих областей современной физики (см., например, монографии [1], [2]). Одной из базовых моделей туннелирования является одномерное движение в потенциальном поле, имеющем вид двойной ямы, в общем случае несимметричной. Задача об аналитическом изучении спектра и волновых функций в случае потенциала с двумя ямами имеет богатую историю: первые результаты, носящие качественный характер, содержались еще в работе 1927 года [3].

* Московский институт электроники и математики при Национальном исследовательском университете "Высшая школа экономики", Москва, Россия.

E-mail: evgeniy.bora@gmail.com 


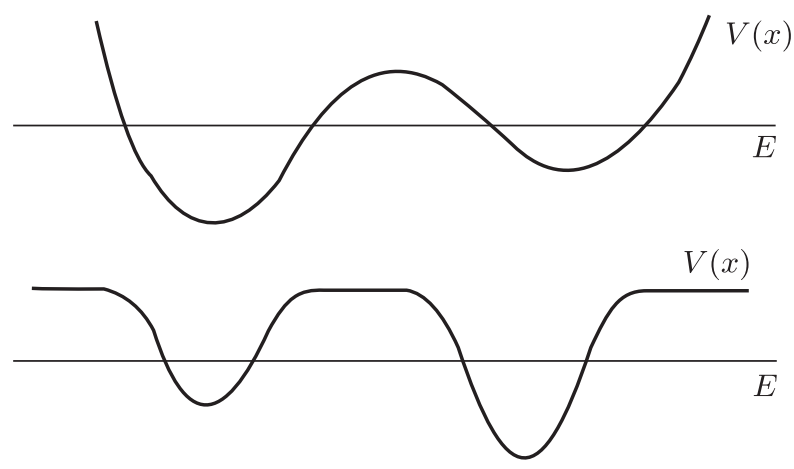

Рис. 1

Мы рассматриваем квазиклассическую асимптотику волновых функций стационарного одномерного уравнения Шредингера

$$
\left(-\hbar^{2} \frac{d^{2}}{d x^{2}}+V(x)\right) \psi=E \psi, \quad 0<\hbar \ll 1,
$$

где $\hbar$ - малый параметр квазиклассического приближения.

Предполагается, что гладкий вещественный потенциал $V(x)$ имеет вид двойной ямы (см. рис. 1), т. е. классическая область движения частицы $(V(x)<E)$ состоит из двух конечных интервалов. Между двумя ямами находится потенциальный барьер, и энергия $E$ не близка к вершине потенциального барьера. Пусть спектр энергий вблизи $E$ является дискретным. Известно, что в случае зеркально-симметричного потенциала этот спектр состоит из пар экспоненциально близких точек, а соответствующие собственные функции являются симметричными и антисимметричными [4]. Это приводит к эффекту туннельной транспортации, когда частица, находящаяся в начальный момент в одной яме, через некоторый промежуток времени может быть обнаружена в другой яме с вероятностью, близкой к единице.

Аналог этого результата для симметричной ямы имеет место и в многомерном случае: известна асимптотика расщепления нижнего энергетического уровня [5].

В настоящей работе мы изучаем случай несимметричного потенциала $V(x)$. Такие потенциалы представляют интерес с физической точки зрения (см. [1], [6]). В несимметричном случае задача существенно усложняется. Однако численные расчеты по-прежнему демонстрируют существование двойной локализации собственных функций [1], [7], [8].

Рассматривая численно решенные примеры, мы замечаем, что вблизи энергии $E$ спектр оператора с двухъямным потенциалом с некоторой точностью состоит из точек $E_{\ell}$ и $E_{\mathrm{r}}$, соответствующих спектрам операторов Шредингера в левой и правой потенциальных ямах в отдельности. Пусть $\psi_{\ell, \mathrm{r}}-$ волновые функции, локализованные в левой и правой потенциальных ямах соответственно. Если $E_{\ell}$ и $E_{\mathrm{r}}$ оказываются близкими, то в спектре оператора с двухъямным потенциалом присутствует пара близких точек, или, как говорят, происходит квазивырождение энергетического уровня. В этом случае соответствующие собственные функции в первом приближении имеют вид линейных комбинаций функций $\psi_{\ell}$ и $\psi_{\mathrm{r}}$. 
Один из методов, при помощи которых можно найти величину расщепления и определить вид собственных функций в случае квазивырождения, основан на рассмотрении сужения оператора $\widehat{H}$ на подпространство, образованное двумя векторами $\psi_{\ell}$ и $\psi_{\mathrm{r}}$, и последующей диагонализации оператора $\widehat{H}$. Этот метод, называемый двухуровневая аппроксимация, широко используется в задачах квантовой механики (см. ссылки в монографии [2]). В данном методе производится вычисление матричных элементов $\left\langle\widehat{H} \psi_{i}, \psi_{j}\right\rangle$ для $i, j=\ell, \mathrm{r}$; искомая величина расщепления находится как расстояние между собственными значениями данной матрицы, а ее собственные векторы дают приближение для коэффициентов линейного разложения собственных векторов оператора $\widehat{H}$ по системе собственных функций $\psi_{\ell, \mathrm{r}}$.

Поскольку подпространство, на которое происходит сужение, не является инвариантным, данный метод дает лишь приближенный результат, точность которого сильно зависит от того, насколько близко указанное подпространство к инвариантному, т. е. от выбора $\psi_{\ell}$ и $\psi_{\mathrm{r}}$. Общее математическое обоснование метода содержится в работах [9], [10]. Этот метод в несколько переработанном виде изложен в приложении к настоящей статье вместе с другими полезными теоремами из теории линейных операторов. Различные вариации данного метода применялись к вычислению туннельного расщепления уровней энергии в несимметричном случае в работах [10]-[13].

В работах [10], [11] предлагается в качестве $\psi_{\ell}, E_{\ell}$ выбирать решение уравнений Шредингера в окрестности $\Omega_{\ell}$ левой ямы, которая не пересекается с правой ямой, и ставить условия Дирихле на границе области $\Omega_{\ell}$. Волновая функция $\psi_{\mathrm{r}}$ и энергия $E_{\mathrm{r}}$ выбираются аналогично. Показано, что при таком выборе можно получить достаточно точные выражения для энергий исходного уравнения в терминах решения задачи Дирихле для отдельных ям, если рассматривать достаточно большие окрестности $\Omega_{i}, i=\ell, \mathrm{r}$. Эти окрестности должны пересекаться и содержать "центр" потенциального барьера. Данным методом в работе [10] получена асимптотика расщепления пары нижних энергетических уровней в случае многомерного несимметричного потенциала (без явного выражения для амплитуды) в терминах

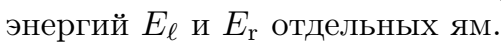

В настоящей работе показано, что такой выбор областей $\Omega_{i}$ является не только достаточным, но и необходимым условием применимости метода двухуровневой аппроксимации (см. разделы 6 и 7). Следовательно, как показано в разделе 7, выбор функций $\psi_{\ell, \mathrm{r}}$, использованный в работе [13], приводит к неверному результату, поскольку области $\Omega_{i}$ выбраны непересекающимися. Одновременно мы отмечаем неточность работы [12], состоящую в том, что для определения центра несимметричного барьера использовалось обычное расстояние на прямой. На самом деле центр потенциального барьера необходимо определять с точки зрения действия по инстантону ${ }^{1)}$.

Кроме вычисления расщепления уровней энергии, метод двухуровневой аппроксимации позволяет определить коэффициенты приближенного разложения собственных функций оператора $\widehat{H}$ по функциям $\psi_{\ell}$ и $\psi_{\mathrm{r}}$. Поскольку собственные функции определены с точностью до нормирующего множителя и ортогональны, достаточно знать асимптотику вероятностей $P_{\ell}(\hbar)$ и $P_{\mathrm{r}}(\hbar)$ обнаружить частицу в левой и правой ямах для одной из пары собственных функций. Волновая функция называется билокализованной, если обе вероятности $P_{i}(\hbar)$ существенно отличны от

\footnotetext{
1) Или в смысле метрики Агмона [10], [14], [15].
} 
нуля при достаточно малых $\hbar$, т. е. $P_{i}(\hbar)=p_{i}+O(\hbar)$ и $p_{i}>0$. Несложно показать, что если одна собственная функция билокализованна, то билокализованна и вторая. Эффект билокализации собственных функций тесно связан с величиной туннельного расщепления уровней энергии.

В работе [16] для двух нижних энергетических уровней в многомерном потенциале доказано, что если обе вероятности $P_{\ell, \mathrm{r}}(\hbar)$ имеют положительный нижний предел при $\hbar \rightarrow 0$, то для величины расщепления $\Delta$ справедлива оценка $\Delta=O\left(e^{-S / \hbar}\right)$. В работе [10] эта оценка уточнена в случае пары нижних энергетических уровней.

Основной результат настоящей работы состоит в доказательстве критерия двойной локализации собственных функций (теорема 1), демонстрирующего связь амплитуды величины расщепления уровней энергии и вероятностей $p_{\ell}$ и $p_{\mathrm{r}}$. В теореме также доказана связь с формулой для расщепления уровней энергии в терминах решения спектральной задачи для правой и левой ямы по отдельности (формула в духе работы [10]). Соответствующие формулы для расщепления имеют вид

$$
\begin{aligned}
& \Delta=\frac{\delta}{2 \sqrt{p_{\ell} p_{\mathrm{r}}}}(1+O(\hbar)), \\
& \Delta=\sqrt{\left(E_{\mathrm{r}}-E_{\ell}\right)^{2}+\delta^{2}}(1+O(\hbar)) ;
\end{aligned}
$$

здесь

$$
\delta=2 \hbar^{2}\left(\psi_{\ell} \frac{d \psi_{\mathrm{r}}}{d x}-\psi_{\mathrm{r}} \frac{d \psi_{\ell}}{d x}\right)_{x=c},
$$

где $c$ - центр потенциального барьера с точки зрения инстантонного действия.

Для высоких энергетических уровней (см. раздел 2) получена следующая асимптотическая формула для $\delta$ :

$$
\delta=\hbar \frac{\sqrt{\omega_{\ell} \omega_{\mathrm{r}}}}{\pi} \exp \left(-\frac{1}{\hbar} \int_{x_{\ell}}^{x_{\mathrm{r}}}|p| d x\right)(1+O(\hbar)) .
$$

В случае низких энергетических уровней формула для $\delta$ приведена в разделе 3 .

Для высоких энергетических уровней полученный результат согласуется с результатом, приведенным в книге [4], при наличии симметрии потенциала; в случае отсутствия симметрии полученный нами результат является обобщением этого результата.

Для низких энергетических уровней амплитуда расщепления может быть выражена в терминах решения системы в вариациях для инстантона (см. раздел 3). Подобный метод применялся в работе [17] для пары нижних энергий оператора Шредингера с многомерным симметричным потенциалом.

В разделе 6 рассмотрена задача о туннельном возмущении дискретного спектра оператора Шредингера с потенциалом, имеющим вид одной ямы. Задача состоит в оценке возмущения энергии $E_{0}$ оператора Шредингера с потенциалом $V(x)$ при добавлении к нему функции $f(x)$, локализованной только в классически запрещенной зоне. При этом малость $f(x)$ не предполагается. Получена формула для главного члена асимптотики туннельного возмущения:

$$
E-E_{0}=\left\langle f(x) \psi_{0}, \psi_{0}\right\rangle(1+o(1))
$$

где $\psi_{0}$ - волновая функция, соответствующая энергии $E_{0}$. 
В разделе 6 доказано, что задача о туннельном возмущении спектра одноямного потенциала эквивалентна задаче о деформации одной стороны потенциального барьера двухъямного потенциала. При такой деформации, где носитель $f(x)$ лежит между центром потенциального барьера и точкой поворота, происходит разрушение двойной локализации собственных функций, и для расщепления справедлива формула

$$
\Delta=\left\langle f(x) \psi_{i}, \psi_{i}\right\rangle(1+o(1)),
$$

где $i=\ell$ или $i=\mathrm{r}$ при деформации левой или правой стороны барьера.

В работе [18] рассматривалась задача о деформации одной стороны потенциального барьера симметричного двухъямного потенциала для пары нижних энергетических уровней. Было показано, что подобная деформация приводит к разрушению двойной локализации собственных функций, а для величины расщепления справедлива оценка

$$
\Delta=\exp \left(-\frac{2 S+o(1)}{\hbar}\right),
$$

где $S$ - действие по инстантону между точкой поворота и носителем функции $f(x)$. Многомерное обобщение данного результата дано в работе [15].

Из нашей формулы легко следуют оценки, приведенные в работе [18]. Полученная формула позволяет вычислить не только логарифмический предел, но и величину амплитуды расщепления для любой заданной функции $f(x)$.

\section{2. ВЕЛИЧИНА РАСЩЕПЛЕНИЯ И АМПЛИТУДА ТУННЕЛИРОВАНИЯ}

В этом разделе мы рассмотрим случай высоких энергетических уровней. Предположим, что энергия $E$ больше, чем минимумы двухъямного потенциала, и меньше, чем вершина потенциального барьера. Наличие в потенциале двух ям означает, что уравнение $V(x)=E$ имеет четыре простых корня - точки поворота, и $V(x)>E+e$ при достаточно больших $x$ и некотором $e>0$.

Пусть между точками поворота $x_{\ell}$ и $x_{\mathrm{r}}$ находится потенциальный барьер, т. е. $V(x)>E$ при $x_{\ell}<x<x_{\mathrm{r}}$. Определим точку $c$ из равенства

$$
\int_{x_{\ell}}^{c}|p(x)| d x=\int_{c}^{x_{\mathrm{r}}}|p(x)| d x,
$$

где $p(x)=\sqrt{E-V(x)}$. Точка $c$ является центром потенциального барьера с точки зрения инстантонного действия. Пусть точки $a$ и $b$ выбраны так, что

$$
x_{\ell}<a<c<b<x_{\mathrm{r}} .
$$

Введем два гладких потенциала $V_{\ell, \mathrm{r}}$ (см. рис. 2), удовлетворяющих следующим двум условиям:

$$
V_{\ell}(x) \equiv V(x) \quad \text { при } \quad x \leqslant b, \quad V_{\mathrm{r}}(x) \equiv V(x) \quad \text { при } \quad x \geqslant a
$$

и

$$
V_{\ell}(x)>E+e \quad \text { при } \quad x \geqslant b, \quad V_{\mathrm{r}}(x)>E+e \quad \text { при } \quad x \leqslant a
$$

для некоторого $e>0$. 


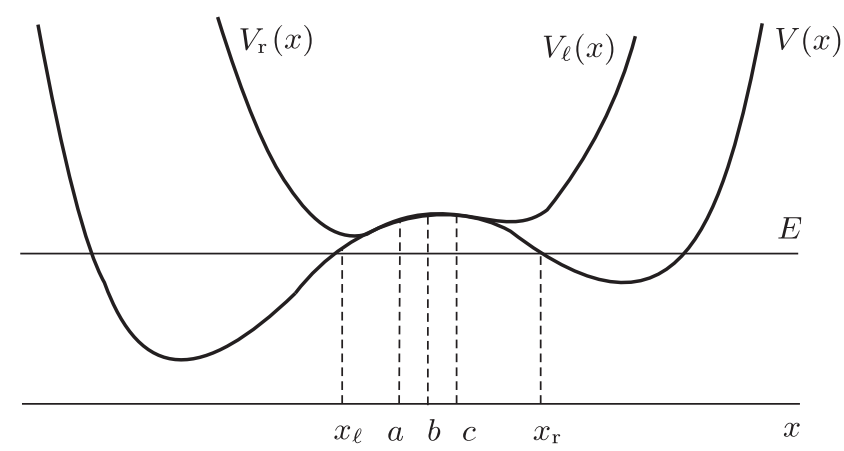

Рис. 2

Определим операторы Шредингера с потенциалами $V_{\ell, \mathrm{r}}$ :

$$
\widehat{H}_{i}=-\hbar^{2} \frac{d^{2}}{d x^{2}}+V_{i}(x), \quad i=\ell, \mathrm{r} .
$$

Потенциалы $V_{\ell, \mathrm{r}}$ являются одноямными для энергии $E$, поскольку классическая область движения частицы в каждом из потенциалов $V_{\ell, \mathrm{r}}(x)$ представляет собой один интервал. Следовательно, для спектров операторов $\widehat{H}_{i}$ справедливы хорошо известные результаты квазиклассического анализа [4], [19], перечисленные ниже.

I. Вблизи энергии $E$ спектр оператора $\widehat{H}_{i}$ является дискретным и невырожденным. Расстояние между соседними точками спектра имеет порядок $\hbar$.

II. Пусть значение $E_{i}$ принадлежит спектру оператора $\widehat{H}_{i}, i=\ell, \mathrm{r}$, и близко к $E$. Пусть $\psi_{i}$ - соответствующая нормированная собственная функция. Тогда равномерно по $x \in[a, b]$ справедливы асимптотические формулы

$$
\begin{aligned}
& \psi_{\ell}(x)=\frac{C_{\ell}}{\sqrt{|p|}} \exp \left(-\frac{1}{\hbar} \int_{x_{\ell}}^{x}|p| d x\right)(1+O(\hbar)), \\
& \psi_{\mathrm{r}}(x)=\frac{C_{\mathrm{r}}}{\sqrt{|p|}} \exp \left(-\frac{1}{\hbar} \int_{x}^{x_{\mathrm{r}}}|p| d x\right)(1+O(\hbar)),
\end{aligned}
$$

причем эту асимптотику можно дифференцировать.

III. Константы нормировки $C_{\ell, \mathrm{r}}$ можно выбрать следующим образом:

$$
C_{i}=\frac{1}{2} \sqrt{\frac{\omega_{i}}{\pi}}, \quad i=\ell, \mathrm{r},
$$

где $\omega_{i}$ - классическая частота колебаний в потенциальной яме $V_{i}(x)$ для энергии $E$.

Результаты I и II носят общий характер и справедливы для произвольного одноямного потенциала, а утверждение III справедливо в случае высоких энергетических уровней.

Операторы $\widehat{H}_{\ell, \mathrm{r}}$ фактически описывают левую и правую потенциальные ямы исходного оператора $\widehat{H}$. Несложно показать (см. ниже доказательство теоремы 1 ), что спектр оператора $\widehat{H}$ вблизи $E$ можно получить с экспоненциальной точностью 
при объединении спектров операторов $\widehat{H}_{\ell}$ и $\widehat{H}_{\mathrm{r}}$. При таком объединении может возникнуть эффект квазивырождения энергетического уровня, когда энергия $E_{\ell}$ экспоненциально близка к энергии $E_{\mathrm{r}}$. Если квазивырождения не происходит, то в качестве приближенной собственной функции оператора $\widehat{H}$ можно взять функцию $\psi_{\ell}$ или $\psi_{\mathrm{r}}$. В случае квазивырождения энергетических уровней в спектре оператора $\widehat{H}$ присутствует пара экспоненциально близких точек спектра, а собственные функции приближенно имеют вид линейных комбинаций функций $\psi_{\ell}$ и $\psi_{\mathrm{r}}$.

Пусть $\psi$ - собственная функция оператора $\widehat{H}$. Вероятности $P_{\ell, \mathrm{r}}(\hbar)$ обнаружить частицу в левой и правой потенциальных ямах могут быть посчитаны как интегралы от $|\psi(x)|^{2}$ при $x<c$ для левой ямы и при $x>c$ для правой ямы. Из условия нормированности волновых функций получаем

$$
P_{\ell}(\hbar)+P_{\mathrm{r}}(\hbar)=1
$$

Следовательно, в качестве величины, которая показывает, где сосредоточена волновая функция, можно взять отношение вероятностей $P_{\mathrm{r}}(\hbar) / P_{\ell}(\hbar)$.

Будем говорить, что волновая функция $\psi$ имеет двойную локализацию, если существует число $\mu>0$ такое, что

$$
\frac{P_{\mathrm{r}}(\hbar)}{P_{\ell}(\hbar)}=\mu^{2}+O(\hbar) .
$$

Тогда вероятности $P_{\ell, \mathrm{r}}(\hbar)$ обнаружить частицу как в левой, так и в правой потенциальной ямах существенно отличны от нуля, и для них справедливы асимптотические формулы

$$
\begin{aligned}
& P_{\ell}(\hbar)=p_{\ell}(1+O(\hbar))=\frac{1}{1+\mu^{2}}(1+O(\hbar)) \\
& P_{\mathrm{r}}(\hbar)=p_{\mathrm{r}}(1+O(\hbar))=\frac{\mu^{2}}{1+\mu^{2}}(1+O(\hbar)) .
\end{aligned}
$$

От значения величины $\mu$ во многом зависит динамика частицы в потенциале с двумя ямами, как будет показано ниже в разделе 4.

Положим

$$
\delta(\hbar)=4 \hbar C_{\ell} C_{\mathrm{r}} \exp \left(-\frac{1}{\hbar} \int_{x_{\ell}}^{x_{\mathrm{r}}}|p| d x\right) .
$$

Величина $\delta(\hbar)$ является характерным масштабом экспоненциальной малости для туннельных эффектов в двухъямном потенциале.

В следующей теореме сформулирован критерий резонансного туннелирования и установлена связь между расщеплением энергетических уровней $\Delta$, амплитудой туннелирования $\mu$ и расстоянием между $E_{\ell}$ и $E_{\mathrm{r}}$.

Теорема 1. Для фиксированного неотрицательного числа $\lambda$ следующие три условия эквивалентны:

1. Вблизи энергии Е у оператора $\widehat{H}$ существует билокализованная собственная функция, и для амплитудь туннелирования справедлива формула

$$
\mu=\sqrt{1+\lambda^{2}} \pm \lambda .
$$


2. Вблизи энергии Е в спектре оператора $\widehat{H}$ существует пара экспоненциально близких точек, расстояние между которыми задается асимптотической формулой

$$
\Delta=\sqrt{1+\lambda^{2}} \delta(\hbar)(1+O(\hbar))
$$

3. Для расстояния между точками $E_{i}$ верна асимптотическая формула

$$
\left|E_{\mathrm{r}}-E_{\ell}\right|=\delta(\hbar)(\lambda+O(\hbar)) \text {. }
$$

Перед доказательством теоремы приведем ряд замечаний.

Данная теорема обобщает результаты симметричного случая. Очевидно, в случае симметричного потенциала выполнены все три условия и $\lambda=0$. В этом случае для расщепления справедлива асимптотическая формула Ландау-Лифшица

$$
\Delta=\frac{\omega \hbar}{\pi} \exp \left(-\frac{1}{\hbar} \int_{x_{\ell}}^{x_{\mathrm{r}}}|p| d x\right)(1+O(\hbar)) .
$$

В общем случае число $\lambda$, как и $\mu$, количественно характеризует двойную локализацию собственных функций. Число $\lambda$ однозначно выражается через $\mu$, поскольку в формуле из условия 1 знак плюс берется, если $\mu>1$, а знак минус берется, если $\mu<1$. Для каждого фиксированного $\lambda$ существуют два значения $\mu=\mu_{1,2}$, при этом $\mu_{1}=\mu_{2}^{-1}$. Два разных значения $\mu$ отвечают паре собственных функций, соответствующих паре близких собственных значений оператора $\widehat{H}$. Следовательно, из существования одной билокализованной собственной функции с $\mu=\mu_{1}$ следует наличие второй собственной функции с обратным показателем $\mu=\mu_{2}=\mu_{1}^{-1}$. Если одна собственная функция больше локализована в левой яме $\left(\mu_{1}<1\right)$, то другая больше локализована в правой $\left(\mu_{2}>1\right)$, и наоборот.

Если $\lambda=0$, то $\mu=1$, и имеет место туннельная транспортация (см. раздел 4). При $\lambda=0$ для величины расщепления справедлива асимптотическая формула

$$
\Delta=\hbar \frac{\sqrt{\omega_{\ell} \omega_{\mathrm{r}}}}{\pi} \exp \left(-\frac{1}{\hbar} \int_{x_{\ell}}^{x_{\mathrm{r}}}|p| d x\right)(1+O(\hbar)) .
$$

При произвольном значении $\lambda \geqslant 0$ величина $\delta(\hbar)$ может быть вычислена по формуле

$$
\delta(\hbar)=2 \hbar^{2}\left[\psi_{\ell} \frac{d \psi_{\mathrm{r}}}{d x}-\psi_{\mathrm{r}} \frac{d \psi_{\ell}}{d x}\right]_{x=c},
$$

это напрямую следует из определения величины $\delta$ и асимптотики функций $\psi_{\ell, \mathrm{r}}(x)$.

Исключая константу $\lambda$ из условий 1 и 2 теоремы 1 , можно найти уравнение связи между расщеплением энергетических уровней $\Delta$ и амплитудой туннелирования $\mu$ :

$$
\Delta=\frac{1}{2}\left(\mu+\frac{1}{\mu}\right) \delta(\hbar)(1+O(\hbar)) .
$$

Выражая $\mu$ через $p_{\ell, \mathrm{r}}$ и подставляя выражение для $\delta(\hbar)$, получаем

$$
\begin{aligned}
& \Delta=\frac{\delta(\hbar)}{2 \sqrt{p_{\ell} p_{\mathrm{r}}}}(1+O(\hbar)) \\
& \Delta=\frac{\hbar}{2 \pi} \sqrt{\frac{\omega_{\ell} \omega_{\mathrm{r}}}{p_{\ell} p_{\mathrm{r}}}} \exp \left(-\frac{1}{\hbar} \int_{x_{\ell}}^{x_{\mathrm{r}}}|p| d x\right)(1+O(\hbar)) .
\end{aligned}
$$


Величина $\mu+\mu^{-1}=\left(p_{\ell} p_{\mathrm{r}}\right)^{-1 / 2}$ не зависит от того, для какой из двух собственных функций она вычислена.

Из последних формул хорошо видно, как с ростом расщепления $\Delta$ быстро исчезает двойная локализация собственных функций, т. е. одна из вероятностей $p_{\ell, \mathrm{r}}$ стремится к нулю. Минимальное расщепление $\Delta \approx \delta(\hbar)$ соответствует максимальной билокализации $p_{\ell}=p_{\mathrm{r}}=1 / 2$ и наоборот.

Также отметим, что теорему 1 можно применять для анализа туннелирования и в случае, когда потенциал зависит от $\hbar$. Тогда необходимо пересчитывать величину $\delta(\hbar)$. Такая зависимость может возникнуть неявно при зависимости потенциала от внешнего параметра.

ДокАЗАТЕЛЬСТво теоремы 1 . Пусть $a^{\prime}, b^{\prime}$ не зависят от $\hbar$ и

$$
x_{\ell}<a<a^{\prime}<c<b^{\prime}<b<x_{\mathrm{r}} .
$$

Определим две гладкие срезающие функции $\sigma_{\ell, \mathrm{r}}(x)$ так, что справедливы условия

$$
\sigma_{\ell}(x) \equiv\left\{\begin{array} { l l } 
{ 1 , } & { x \leqslant b ^ { \prime } , } \\
{ 0 , } & { x \geqslant b , }
\end{array} \sigma _ { \mathrm { r } } ( x ) \equiv \left\{\begin{array}{ll}
1, & x \geqslant a^{\prime} \\
0, & x \leqslant a
\end{array}\right.\right.
$$

Тогда пересечение носителей функций $\sigma_{\ell, \mathrm{r}}$ вложено в $[a, b]$ и заведомо содержит отрезок $\left[a^{\prime}, b^{\prime}\right]$, а следовательно, и точку $c$. Из определения $V_{\ell, \text { г }}$ следует, что

$$
\sigma_{i}(x)\left[V(x)-V_{i}(x)\right] \equiv 0, \quad \sigma_{i}(x)\left[\widehat{H}-\widehat{H}_{i}\right]=0, \quad i=\ell, \mathrm{r},
$$

где во втором равенстве $\sigma_{i}(x)$ понимается как оператор умножения на функцию.

Для начала докажем, что справедливость любого из трех условий теоремы влечет наличие квазивырождения, т. е. существование энергий $E_{i}$ в спектре оператора $\widehat{H}_{i}$, $i=\ell, \mathrm{r}$, таких, что расстояние между ними экспоненциально мало.

Пусть справедливо условие 1 и $\psi$ - соответствующая собственная функция оператора $\widehat{H}$, а $E$ - собственное значение. Пусть

$$
u_{i}=\frac{\sigma_{i} \psi}{\sqrt{p_{i}}}, \quad i=\ell, \mathrm{r}
$$

Тогда

$$
\left\|u_{\ell}\right\|^{2}=\frac{1}{p_{\ell}} \int_{-\infty}^{b}\left|\sigma_{\ell} \psi\right|^{2} d x=1+\frac{1}{p_{\ell}} \int_{c}^{b}\left|\sigma_{\ell} \psi\right|^{2} d x \approx 1,
$$

поскольку интеграл по отрезку $[c, b]$ экспоненциально мал. Аналогично имеем

$$
\left\|u_{\mathrm{r}}\right\| \approx 1
$$

Покажем, что $u_{i}$ являются квазимодами для операторов $\widehat{H}_{i}$ с экспоненциальной точностью. Меняя порядок операторов дифференцирования и умножения на функцию $\sigma_{i}(x)$, легко получить выражение для невязки:

$$
\left(\widehat{H}_{i}-E\right) u_{i}=\frac{-\hbar^{2}}{\sqrt{p_{i}}}\left(\sigma_{i}^{\prime \prime}+2 \sigma_{i}^{\prime} \frac{d}{d x}\right) \psi
$$


Поскольку носители функций $\sigma_{i}^{\prime}(x)$ и $\sigma_{i}^{\prime \prime}(x)$ вложены в отрезок $[a, b]$, на котором функция $\psi(x)$ и ее производная экспоненциально малы, получаем, что $u_{i}$ являются квазимодами для $\widehat{H}_{i}$ с экспоненциальной точностью. Таким образом, доказано, что из условия 1 следует квазивырождение спектра.

Пусть справедливо условие 2, существуют экспоненциально расщепленные собственные значения $E_{1,2}$ оператора $\widehat{H}$, а $\psi_{1,2}$ - соответствующие собственные функции. Можно считать, что функции $\psi_{1,2}$ не являются билокализованными, иначе было бы справедливо условие 1 , из которого следует квазивырождение. Функции $\psi_{1,2}$ не могут быть локализованы в одной яме, поскольку это противоречит их ортогональности. Пусть для определенности $\psi_{1}$ локализована в левой яме, а $\psi_{2}-$ в правой яме. Тогда вместо $\psi_{1,2}$ и $E_{1,2}$ будем писать $\psi_{\ell, \mathrm{r}}$ и $E_{\ell, \mathrm{r}}$.

Пусть $u_{i}=\sigma_{i} \psi_{i}$. Тогда $u_{i}$ являются квазимодами для $\widehat{H}_{i}$ с экспоненциальной точностью:

$$
\left\|u_{i}\right\| \approx 1, \quad\left(\widehat{H}_{i}-E_{i}\right) u_{i}=-\hbar^{2}\left(\sigma_{i}^{\prime \prime}+2 \sigma_{i}^{\prime} \frac{d}{d x}\right) \psi_{i} .
$$

Получаем, что квазивырождение следует из условия 2.

Пусть справедливо условие 3. Тогда квазивырождение, очевидно, имеет место, поскольку величина $\delta(\hbar)$ экспоненциально мала.

Мы доказали, что справедливость любого из трех условий теоремы влечет наличие квазивырождения. Предположим, что для некоторого $\lambda$ справедливо хотя бы одно из условий данной теоремы, следовательно, квазивырождение имеет место. Пусть $E_{i}$ - собственные значения оператора $\widehat{H}_{i}$, а $\psi_{i}$ - соответствующие собственные функции, $i=\ell, \mathrm{r}$. Следовательно, величина $\left|E_{\mathrm{r}}-E_{\ell}\right|$ экспоненциально мала.

Введем ортонормированные функции

$$
\begin{gathered}
u_{\ell}(x)=\gamma_{1} \sigma_{\ell}(x) \psi_{\ell}(x), \quad u_{\mathrm{r}}(x)=\gamma_{2}\left(\sigma_{\mathrm{r}}(x) \psi_{\mathrm{r}}(x)+\gamma_{3} \sigma_{\ell}(x) \psi_{\ell}(x)\right), \\
\left\langle u_{i}, u_{j}\right\rangle=\delta_{i j}, \quad i, j=\ell, \mathrm{r} .
\end{gathered}
$$

Учитывая нормированность функций $\psi_{i}(x), i=\ell, \mathrm{r}$, получаем

$$
\gamma_{1,2}=1+O(\hbar)
$$

Из условия ортогональности функций $u_{1}$ и $u_{2}$ имеем

$$
0=\left\langle u_{\ell}, u_{\mathrm{r}}\right\rangle=\gamma_{1} \gamma_{2} \int_{a}^{b} \sigma_{\ell} \sigma_{\mathrm{r}} \psi_{\ell} \psi_{\mathrm{r}} d x+\gamma_{2} \gamma_{3} \gamma_{1}^{-1}, \quad \gamma_{3}=O(\delta(\hbar))
$$

Покажем, что $u_{i}$ являются квазимодами для оператора $\widehat{H}$, вычислив асимптотику невязки:

$$
\begin{aligned}
\left(\widehat{H}-E_{\ell}\right) u_{\ell} & =\left[-\hbar^{2} \frac{d^{2}}{d x^{2}}, \sigma_{\ell}(x)\right] \gamma_{1} \psi_{\ell}(x)+\sigma_{\ell}(x) \gamma_{1}\left(\widehat{H}_{\ell}-E_{\ell}\right) \psi_{\ell}(x)= \\
& =\hbar \sigma_{\ell}^{\prime}(x) 2 C_{\ell} \sqrt{|p|} \exp \left(-\frac{1}{\hbar} \int_{x_{\ell}}^{x}|p| d x\right)(1+O(\hbar)), \\
\left(\widehat{H}-E_{\mathrm{r}}\right) u_{\mathrm{r}} & =-\hbar \sigma_{\mathrm{r}}^{\prime}(x) 2 C_{\mathrm{r}} \sqrt{|p|} \exp \left(-\frac{1}{\hbar} \int_{x}^{x_{\mathrm{r}}}|p| d x\right)(1+O(\hbar)) .
\end{aligned}
$$


Введем обозначение

$$
\varepsilon=\max \left[C_{\ell} \exp \left(-\frac{1}{\hbar} \int_{x_{\ell}}^{b^{\prime}}|p| d x\right), C_{\mathrm{r}} \exp \left(-\frac{1}{\hbar} \int_{a^{\prime}}^{x_{\mathrm{r}}}|p| d x\right)\right] .
$$

Величина $\varepsilon$ является экспоненциально малой и при этом $\varepsilon^{2}$ экспоненциально меньше, чем $\delta(\hbar)$. Получаем оценки

$$
\left\|\left(\widehat{H}-E_{i}\right) u_{i}\right\|=O(\varepsilon), \quad i=\ell, \mathrm{r} .
$$

Следовательно, $u_{\ell}$ и $u_{\mathrm{r}}$ действительно являются квазимодами.

Теперь предположим, что

$$
\left|E_{\mathrm{r}}-E_{\ell}\right|=O(\varepsilon)
$$

Тогда можно воспользоваться теоремой 6 (см. приложение). Ниже будет показано, что если последнее равенство несправедливо, то все три условия теоремы не выполняются.

Введем обозначение $L(\hbar)=\left(E_{\mathrm{r}}-E_{\ell}\right) / \delta$. Величина $L$ введена, как в условии 3 доказываемой теоремы, но выполнение этого условия (существование предела $L(\hbar)$ при $\hbar \rightarrow 0)$ не предполагается. Пусть $E=\left(E_{\ell}+E_{\mathrm{r}}\right) / 2$. Следовательно, имеет место оценка

$$
\left\|(\widehat{H}-E) u_{i}\right\| \leqslant\left\|\left(\widehat{H}-E_{i}\right) u_{i}\right\|+\frac{\left|E_{\mathrm{r}}-E_{\ell}\right|}{2}\left\|u_{i}\right\|=O(\varepsilon), \quad i=\ell, \mathrm{r} .
$$

Таким образом, выполнены все условия теоремы 6. Вычислим матричные элементы $m_{i, j}=\left\langle(\widehat{H}-E) u_{i}, u_{j}\right\rangle, i, j=\ell, \mathrm{r}$ :

$$
\begin{gathered}
m_{\ell, \ell}=\frac{E_{\ell}-E_{\mathrm{r}}}{2}+O\left(\varepsilon^{2}\right), \quad m_{\mathrm{r}, \mathrm{r}}=\frac{E_{\mathrm{r}}-E_{\ell}}{2}+O\left(\varepsilon^{2}\right), \\
m_{\ell, \mathrm{r}}=2 \hbar C_{\ell} C_{\mathrm{r}} \int_{b^{\prime}}^{b} \sigma_{\ell}^{\prime}(x) \exp \left(-\frac{1}{\hbar} \int_{x_{\ell}}^{x_{\mathrm{r}}}|p| d x\right)(1+O(\hbar)) d x=-\frac{\delta}{2}(1+O(\hbar)) .
\end{gathered}
$$

Таким образом,

$$
m=\frac{\delta}{2}\left(\begin{array}{cc}
-L & -1 \\
-1 & L
\end{array}\right)(1+O(\hbar))+O\left(\varepsilon^{2}\right) .
$$

Собственные значения матрицы $m$ равны

$$
\mu_{1,2}= \pm \frac{\delta}{2} \sqrt{1+L^{2}}
$$

Соответствующие собственные векторы имеют вид

$$
\left(\begin{array}{c}
-1 \\
L+\sqrt{1+L^{2}}
\end{array}\right), \quad\left(\begin{array}{c}
L+\sqrt{1+L^{2}} \\
1
\end{array}\right)
$$

где множитель $1+O(\hbar)$ опущен для наглядности. Определим величины

$$
\begin{aligned}
& \widetilde{E}_{1}=E+\mu_{1}=\frac{E_{\mathrm{r}}+E_{\ell}}{2}+\frac{\delta}{2} \sqrt{1+L^{2}} \\
& \widetilde{E}_{2}=E+\mu_{2}=\frac{E_{\mathrm{r}}+E_{\ell}}{2}-\frac{\delta}{2} \sqrt{1+L^{2}} .
\end{aligned}
$$


По теореме 6 для энергий $\widetilde{E}_{i}$ можно построить квазимоды $\tilde{u}_{i}$, для которых

$$
\left\|\left(\widehat{H}-\widetilde{E}_{i}\right) \tilde{u}_{i}\right\|=O\left(\frac{\varepsilon^{2}}{\hbar}\right) .
$$

С точностью до нормировки их главные члены равны

$$
v_{1}=-u_{\ell}+\left(L+\sqrt{1+L^{2}}\right) u_{\mathrm{r}}, \quad v_{2}=\left(L+\sqrt{1+L^{2}}\right) u_{\ell}+u_{\mathrm{r}}
$$

Таким образом, в $O\left(\varepsilon^{2} / \hbar\right)$-окрестностях энергий $\widetilde{E}_{1}$ и $\widetilde{E}_{2}$ имеется по одной точке спектра оператора $\widehat{H}$. Расстояние между этими точками можно оценить как

$$
\Delta=\sqrt{1+L^{2}} \delta(1+O(\hbar))+O\left(\frac{\varepsilon^{2}}{\hbar}\right)=\sqrt{1+L^{2}} \delta(1+O(\hbar)) .
$$

Величину $O\left(\varepsilon^{2} / \hbar\right)$ можно отбросить, так как отношение $\varepsilon^{2} / \delta$ экспоненциально мало. Малость $\varepsilon^{2} / \delta$ обеспечивается выбором точки $c$.

Из теоремы 5 (см. приложение) следует, что для собственной функции $\phi_{i}$ оператора $\widehat{H}$, соответствующей точке спектра, близкой к $\widetilde{E}_{i}$, справедлива асимптотическая формула

$$
\phi_{i}=v_{i}(x)+O\left(\hbar^{\infty}\right) .
$$

Следовательно, $\phi_{i}$ являются билокализованными, только если существует число $\lambda$ такое, что

$$
L(\hbar)=\lambda+O(\hbar)
$$

Тогда величина $\mu$ для $\phi_{1}$ записывается как

$$
\mu=\sqrt{1+\lambda^{2}}-\lambda
$$

а для $\phi_{2}$ мы имеем

$$
\mu=\sqrt{1+\lambda^{2}}+\lambda
$$

Полученные результаты справедливы в предположении, что выполнено одно из условий теоремы и $\left|E_{\mathrm{r}}-E_{\ell}\right|=O(\varepsilon)$. Для доказательства эквивалентности всех трех условий покажем, что из условия 3 следуют условия 1 и 2 и обратно.

Пусть выполнено условие 3 , т. е. $L(\hbar)=\lambda+O(\hbar)$. Из полученных формул для $\mu$ и $\Delta$ следует справедливость условий 1 и 2.

Пусть выполнено условие 1 с некоторым $\lambda$. Поскольку $\lambda$ однозначно определяется числом $\mu$ (напомним, что $\lambda \geqslant 0)$, имеем

$$
|L(\hbar)|=\frac{\left|E_{\mathrm{r}}-E_{\ell}\right|}{\delta(\hbar)}=\lambda+O(\hbar),
$$

следовательно, выполнено условие 3.

Аналогично из условия 2 вытекает условие 3, поскольку при справедливости условия 2 величина $\lambda$ однозначно определяется отношением $\Delta / \delta$.

Все предыдущие рассуждения опирались на предположение, что

$$
\left|E_{\mathrm{r}}-E_{\ell}\right|=O(\varepsilon) \text {. }
$$


Покажем, что если это не так, то не выполнено ни одно из трех условий теоремы. Условие 3 не выполнено, так как оно влечет $\left|E_{\mathrm{r}}-E_{\ell}\right|=O(\varepsilon)$.

Пусть $u_{i}=\sigma_{i}(x) \psi_{i}(x), i=\ell, \mathrm{r}$. Тогда

$$
\left\|\left(\widehat{H}-E_{i}\right) u_{i}\right\|=O(\varepsilon), \quad i=\ell, \mathrm{r} .
$$

Из теоремы 4 (см. приложение) следует, что существует собственное значение $\widetilde{E}_{i}$ оператора $\widehat{H}$ такое, что

$$
\left|E_{i}-\widetilde{E}_{i}\right|=O(\varepsilon), \quad i=\ell, \mathrm{r}
$$

Соответствующие собственные функции имеют асимптотику (см. теорему 5)

$$
\tilde{\psi}_{i}=u_{i}+O\left(\varepsilon \Delta^{-1}\right)
$$

где $\Delta$ - расстояние между двумя близкими точками спектра оператора $\widehat{H}$. Очевидна оценка

$$
\Delta=\left|E_{\ell}-E_{\mathrm{r}}\right|+O(\varepsilon) .
$$

Следовательно, отношение $\Delta / \delta$ неограниченно, и условие 2 не выполнено.

Предположим, что имеет место двойная локализация. Из двойной локализации следует существование предела $\mu^{2} \neq 0$ для отношения вероятностей $P_{\mathrm{r}}(\hbar) / P_{\ell}(\hbar)$. Поскольку отношение $\Delta / \varepsilon$ неограниченно, функции $\tilde{\psi}_{i}$ будут близки к $u_{i}$ при некотором малом $\hbar$. Получаем, что предел отношения вероятностей, если он существует, равен либо нулю, либо бесконечности, что противоречит определению двойной локализации. Теорема полностью доказана.

\section{3. СЛУЧАЙ ЭНЕРГИИ, БЛИЗКОЙ К МИНИМУМУ ПОТЕНЦИАЛА}

Аналог теоремы 1 справедлив для нижних энергетических уровней. Необходимо только пересчитать величины $C_{i}$ и соответственно изменить $\delta(\hbar)$. Следует разделять случаи, когда оба локальных минимума потенциала $V(x)$ соответствуют одной энергии и когда значения $V(x)$ в локальных минимумах не совпадают. Для примера рассмотрим случай совпадения значений $V(x)$ в точках локальных минимумов.

Пусть $\xi_{1,2}$ - координаты двух невырожденных локальных минимумов двухъямного потенциала $V(x)$. Будем считать, что

$$
V\left(\xi_{i}+x\right)=\omega_{i}^{2} x^{2}(1+O(x)), \quad i=1,2 .
$$

Тогда для величины $\delta$, если $n$-й уровень $E_{\ell}^{(n)}$ левой ямы близок к $m$-му уровню $E_{\mathrm{r}}^{(m)}$ правой ямы, справедлива асимптотическая формула

$$
\delta=4 \hbar \frac{\sqrt{\omega_{1} \omega_{2}}}{\sqrt{\pi n ! m !}}\left(\frac{2}{\hbar}\right)^{(n+m) / 2} J_{1}^{n+1 / 2} J_{2}^{m+1 / 2} \exp \left(-\frac{1}{\hbar} \int_{\xi_{1}}^{\xi_{2}} \sqrt{V(x)} d x\right)(1+O(\hbar))
$$

где

$$
\begin{aligned}
& J_{1}=\sqrt{\omega_{1}} \lim _{t \rightarrow \xi_{1}+0}\left\{\left(t-\xi_{1}\right) \exp \left(\omega_{1} \int_{t}^{c} \frac{d x}{\sqrt{V(x)}}\right)\right\}, \\
& J_{2}=\sqrt{\omega_{2}} \lim _{t \rightarrow \xi_{2}-0}\left\{\left(\xi_{2}-t\right) \exp \left(\omega_{2} \int_{c}^{t} \frac{d x}{\sqrt{V(x)}}\right)\right\} .
\end{aligned}
$$


Если выполнены условия теоремы 1 , то $\left|E_{\mathrm{r}}^{(m)}-E_{\ell}^{(n)}\right|$ экспоненциально мало, следовательно, совпадают первые члены разложения по $ћ$ данных энергий:

$$
\omega_{1}(2 n+1)=\omega_{2}(2 m+1)
$$

Таким образом, отношение частот колебаний классической частицы в левой и правой ямах есть рациональное число. В связи с этим возникновение туннельных эффектов в двухъямном потенциале часто называют резонансным туннелированием. Данный термин не вполне удачен, поскольку рациональность отношения частот является лишь слабым необходимым условием, и из нее не следует возникновение таких эффектов, как туннельная транспортация и двойная локализация собственных функций.

Следуя методу работы [17], выразим пределы $J_{1}$ и $J_{2}$ через асимптотику решения системы в вариациях.

Рассмотрим гамильтонову систему, определяющую инстантон,

$$
\dot{q}=2 p, \quad \dot{p}=V^{\prime}(q),
$$

с граничными условиями $q(-\infty)=\xi_{1}, q(0)=c, q(\infty)=\xi_{2}$. Эта система получается при изменении знака потенциала. Инстантон можно задать неявной формулой

$$
2 t=\int_{c}^{q} \frac{d x}{\sqrt{V(x)}}
$$

Рассмотрим соответствующую систему в вариациях:

$$
\begin{gathered}
\ddot{z}=2 V^{\prime \prime}(q(t)) z, \\
z( \pm \infty)=0, \quad z(0)=1 .
\end{gathered}
$$

Ее решение имеет вид

$$
z(t)=\sqrt{\frac{V(q(t))}{V(c)}} .
$$

Найдем асимптотику решения системы в вариациях при $t \rightarrow-\infty$ :

$$
z(t) \sim \frac{1}{\sqrt{V(c)}} \omega_{1}\left(q-\xi_{1}\right)
$$

Из определения $J_{1}$ следует, что

$$
q-\xi_{1} \sim J_{1} \omega_{1}^{-1 / 2} \exp \left(\omega_{1} \int_{c}^{q} \frac{d x}{\sqrt{V(x)}}\right), \quad z(t) \sim J_{1} \frac{\sqrt{\omega_{1}}}{\sqrt{V(c)}} e^{2 \omega_{1} t}
$$

Аналогично найдем асимптотику решения системы в вариациях при $t \rightarrow \infty$ :

$$
z(t) \sim J_{2} \frac{\sqrt{\omega_{2}}}{\sqrt{V(c)}} e^{-2 \omega_{2} t}
$$

Таким образом, найдена связь пределов $J_{1,2}$ с асимптотикой решения системы в вариациях для инстантона. 


\section{4. ДИНАМИКА ЧАСТИЦЫ В СЛУЧАЕ РЕЗОНАНСНОГО ТУННЕЛИРОВАНИЯ}

Рассмотрим оператор

$$
\widehat{H}=-\hbar^{2} \frac{d^{2}}{d x^{2}}+V(x)
$$

в котором $V(x)$ является гладкой вещественной функцией, имеющей вид двойной потенциальной ямы.

Рассмотрим динамику частицы в двухъямном потенциале при энергии, близкой к паре квазивырожденных стационарных энергетических уровней. Пусть справедливы условия теоремы 1 , два энергетических уровня $E_{1,2}$ оператора $\widehat{H}$ находятся экспоненциально близко друг к другу, а соответствующие собственные функции $\psi_{1,2}$ билокализованны.

Учитывая условие ортогональности для функций $\psi_{i}$, получаем (см. доказательство теоремы 1)

$$
\psi_{1}=u_{\ell}+\mu u_{\mathrm{r}}+O\left(\hbar^{\infty}\right), \quad \psi_{2}=\mu u_{\ell}-u_{\mathrm{r}}+O\left(\hbar^{\infty}\right),
$$

где $u_{\ell, \mathrm{r}}$ сосредоточены только в левой и правой потенциальных ямах соответственно.

Рассмотрим задачу Коши с начальным состоянием, локализованным в левой яме:

$$
i \hbar \frac{\partial u}{\partial t}=\widehat{H} u,\left.\quad u\right|_{t=0}=u_{\ell} .
$$

Решение данной задачи Коши с точностью $O\left(\hbar^{\infty}\right)$ имеет вид

$$
u=\frac{1}{1+\mu^{2}}\left(e^{-i E_{1} t / \hbar} \psi_{1}+\mu e^{-i E_{2} t / \hbar} \psi_{2}\right) .
$$

Подставляя выражения для $\psi_{1}$ и $\psi_{2}$, получаем

$$
\begin{aligned}
u & =\frac{e^{-i E_{1} t / \hbar}}{1+\mu^{2}}\left(u_{\ell}+\mu u_{\mathrm{r}}+e^{-(i / \hbar)\left(E_{2}-E_{1}\right) t} \mu\left(\mu u_{\ell}-u_{\mathrm{r}}\right)\right)= \\
& =\frac{e^{-i E_{1} t / \hbar}}{1+\mu^{2}}\left(\left(\mu^{2} e^{-(i / \hbar)\left(E_{2}-E_{1}\right) t}+1\right) u_{\ell}+\mu\left(1-e^{-(i / \hbar)\left(E_{2}-E_{1}\right) t}\right) u_{\mathrm{r}}\right) .
\end{aligned}
$$

Полная туннельная транспортация означает, что существует такой момент времени $t=T$, что решение сосредоточено в иной яме, чем та, в которой сосредоточено начальное условие. Следовательно, полная транспортация имеет место, если коэффициент перед $u_{\ell}$ обращается в нуль. Учитывая, что $\mu$ - положительное число, получаем $\mu=1$. При положительных значениях $\mu \neq 1$ возникает только частичная транспортация.

Видно, что туннельная транспортация представляет собой периодический процесс, и его полупериод $T$ - это время, за которое происходит перенос частицы в другую потенциальную яму. Время $T$ экспоненциально велико и имеет вид

$$
T=\frac{\pi \hbar}{E_{2}-E_{1}} .
$$

Используя формулы для $\mu$ и $\Delta$ из теоремы 1 , для максимальной вероятности обнаружить частицу в правой яме получаем

$$
\max _{t} P_{\mathrm{r}}(t)=\left(\frac{\delta}{\Delta}\right)^{2}+O\left(\hbar^{\infty}\right) .
$$




\section{5. ПРИМЕРЫ НЕСИММЕТРИЧНОЙ ТРАНСПОРТАЦИИ}

Классическим примером двухъямного потенциала является полином четвертого порядка. Точное решение для этого примера было изучено в работе [7] с помощью специальных функций.

Рассмотрим оператор $\widehat{H}$ с потенциалом

$$
V(x, s)=(x-1)^{2}(x+1)^{2}+s x,
$$

где $s$ - параметр, характеризующий асимметрию. Будем исследовать спектр оператора $\widehat{H}$ вблизи $E$, где $0<E<1$. Параметр $s$ меняется в пределах $\left(0, s_{0}\right)$, где $s_{0}=s_{0}(E)$ выбрано так, что уравнение $V\left(x, s_{0}\right)=E$ имеет четыре простых корня. Следовательно, рассматривается случай высоких энергетических уровней.

Как и ранее в разделе 2, введем операторы $\widehat{H}_{i}$, константы $c=c(s)$ и $\delta=\delta(s, \hbar)$. Пусть $E_{i}^{(n)}$ - точки спектра оператора $\widehat{H}_{i}, i=\ell, \mathrm{r}$. Квантовые числа $n=n(\hbar)$ выбираются так, что $E_{i}^{(n)}$ близки к $E$. При фиксированном малом $\hbar$ энергетические уровни $E_{i}^{(n)}$ и соответствующие им собственные функции непрерывно зависят от $s$.

При $s=0$ оператор $\widehat{H}_{\ell}$ отличается от $\widehat{H}_{\mathrm{r}}$ заменой $x$ на $-x$. Отсюда $E_{\ell}^{(n)}=E_{\mathrm{r}}^{(n)}$. Из теоремы 1 следует билокализация собственных функций. При увеличении параметра $s$ энергетический уровень $E_{\ell}^{(n)}$ убывает, а $E_{\mathrm{r}}^{(n)}$ возрастает с точностью $O\left(\hbar^{2}\right)$. Это следует из того, что они приближенно удовлетворяют правилу дискретизации Планка-Бора-Зоммерфельда [4]. Из непрерывной зависимости от $s$ следует существование $s_{1}$ такого, что

$$
E_{\ell}^{(n+1)}=E_{\mathrm{r}}^{(n)} \quad \text { при } \quad s=s_{1} .
$$

При $s=s_{1}$ собственные функции оператора $\widehat{H}$, отвечающие собственным значениям с номерами $2 n$ и $2 n+1$, билокализованны, и имеет место транспортация. Аналогичный пример можно рассмотреть и для случая низких энергий.

\section{6. ТУННЕЛЬНОЕ ВОЗМУЩЕНИЕ СПЕКТРА}

В данном разделе мы найдем асимптотику возмущения дискретного спектра оператора Шредингера с одноямным потенциалом при добавлении к нему возмущающего потенциала, который полностью сосредоточен вне области движения классической частицы. Рассмотрение ведется в окрестности определенной энергии. Очевидно, что возмущение спектра окажется экспоненциально малым даже для немалого возмущающего потенциала (для рассматриваемой энергии). Основная цель состоит в получении главного члена асимптотики. Предполагается, что при добавлении возмущения потенциал остается одноямным для заданной энергии.

Пусть задан исходный одноямный потенциал $V(x)$ и соответствующий ему оператор $\widehat{H}_{0}$. Пусть $E_{0}$ - собственное значение оператора $\widehat{H}_{0}$, а $\psi_{0}$ - соответствующая волновая функция. Пусть $\left(x_{1}, x_{2}\right)$ - область классического движения, т. е. $V(x)<E_{0}$ при $x \in\left(x_{1}, x_{2}\right)$. Добавим к исходному оператору возмущение, имеющее вид непрерывной функции $f(x)$, локализованной полностью в классически запрещенной области, а именно

$$
\widehat{H}=\widehat{H}_{0}+f(x), \quad \operatorname{supp} f(x)=[a, b], \quad x_{2}<a<b .
$$


Для волновой функции $\psi_{0}(x)$ при $x \in[a, b]$ справедливо разложение ВКБ

$$
\psi_{0}(x)=\frac{C}{\sqrt{|p|}} \exp \left(-\frac{1}{\hbar} \int_{x_{2}}^{x}|p| d x\right)(1+O(\hbar)) .
$$

Следовательно,

$$
\left\|\left(\widehat{H}-E_{0}\right) \psi_{0}\right\|^{2}=\left\|f(x) \psi_{0}\right\|^{2}=\int_{a}^{b} f^{2}(x) \psi_{0}^{2}(x) d x=\varepsilon^{2},
$$

и для $\varepsilon$ справедлива оценка

$$
\varepsilon \leqslant C_{1} e^{-S / \hbar},
$$

где $S$ - действие по инстантону от точки поворота до носителя функции $f(x)$ :

$$
S=\int_{x_{2}}^{a} \sqrt{V(x)-E} d x .
$$

Из данной оценки получаем, что у оператора $\widehat{H}$ есть точка спектра $E$, расстояние от которой до $E_{0}$ экспоненциально мало (не превосходит $\varepsilon$ ). Если после добавления $f(x)$ потенциал остается одноямным для энергий, близких к $E_{0}$, то $\psi_{0}$ может служить приближением для собственной функции оператора $\widehat{H}$ (см. теорему 5), поскольку у оператора с одноямным потенциалом расстояние между соседними уровнями имеет порядок $\hbar$.

Найдем следующий член разложения $E$ по $\varepsilon$. Поскольку функция $f(x)$ не предполагается малой, формулы обычной теории возмущений неприменимы. Имеет место только слабая оценка $\left|E-E_{0}\right| \leqslant \varepsilon$.

Идея построения асимптотики энергии $E$ состоит в применении теории возмущений для поиска спектра оператора $\widehat{H}$, где в качестве невозмущенного оператора рассматривается не оператор $\widehat{H}_{0}$, а некоторый специально построенный оператор. Данные идеи близки к идеям, использованным при доказательстве теоремы 6.

Tеорема 2. Пусть $f(x) \rightarrow 0$ при $x \rightarrow a$. Тогда для энергии Е оператора $\widehat{H}$ справедлива асимптотическая формула

$$
E-E_{0}=\left\langle f(x) \psi_{0}, \psi_{0}\right\rangle(1+o(1)) .
$$

ДокАзАтЕльСтво. Пусть $\Pi$ - проектор на $\psi_{0}$, а $\Pi^{\prime}=1-\Pi$. Рассмотрим операторы

$$
A=\widehat{H}_{0}+\Pi^{\prime} f(x) \Pi^{\prime}, \quad B=f(x) \Pi+\Pi f(x)-\Pi f(x) \Pi .
$$

Для них справедливы соотношения

$$
\begin{gathered}
A+B=\widehat{H}_{0}+f(x)=\widehat{H}, \quad\|B\|=O(\varepsilon), \\
A \psi_{0}=E_{0} \psi_{0}, \quad B \psi_{0}=f(x) \psi_{0} .
\end{gathered}
$$

Если предположить, что потенциал $V+f(x)$ остается одноямным для энергий, близких к $E_{0}$, то у оператора $\widehat{H}$ существует единственная точка спектра $E$, экспоненциально близкая к $E_{0}$, а остальные точки спектра находятся на расстоянии порядка $\hbar$. 
Следовательно, для поиска асимптотики $E$ можно применить формулу теории возмущений для изолированной точки спектра:

$$
E=E_{0}+\left\langle B \psi_{0}, \psi_{0}\right\rangle+\sum_{k=1}^{+\infty} \frac{\left\langle B \psi_{0}, \phi_{k}\right\rangle^{2}}{E_{0}-\lambda_{k}}+O\left(\frac{\varepsilon^{3}}{\hbar^{2}}\right),
$$

где $\psi_{0}, \phi_{1}, \phi_{2}, \ldots$ - ортонормированный набор собственных функций оператора $A$, а $E_{0}, \lambda_{1}, \lambda_{2}, \ldots$ - соответствующие собственные значения. Члены суммы, для которых энергия $\lambda_{k}$ близка к $E_{0}$, не дают вклада в асимптотику, поскольку для них функции $\phi_{k}$ экспоненциально малы на носителе функции $f(x)$. Следовательно,

$$
E=E_{0}+\left\langle f(x) \psi_{0}, \psi_{0}\right\rangle+O\left(\varepsilon^{2}\right) .
$$

Остается доказать, что

$$
\varepsilon^{2}=\left\langle f(x) \psi_{0}, f(x) \psi_{0}\right\rangle=o\left(\left\langle f(x) \psi_{0}, \psi_{0}\right\rangle\right) .
$$

Данные оценки верны, поскольку вклад в интеграл $\left\langle f(x) \psi_{0}, f(x) \psi_{0}\right\rangle$ дает только малая окрестность точки $a$ и функция $f(x)$ мала в этой окрестности.

Из теоремы 2 можно получить несколько важных следствий.

СлеДСТвиЕ 1. Формула для главного члена асимптотики совпадает с формулой классической теории возмущений, но величина поправки может быть много больше величины малого параметра в и существенно зависит от того, как функиия $f(x)$ стремится $к$ нулю при $x \rightarrow a$.

СлЕДСТВиЕ 2. Справедлива оценка

$$
E-E_{0}=\exp \left(-\frac{2 S+o(1)}{\hbar}\right) .
$$

Простая теория возмущений дает значительно более слабую оценку

$$
\left|E-E_{0}\right| \leqslant \varepsilon=\exp \left(-\frac{S+o(1)}{\hbar}\right)
$$

и не позволяет вычислить порядок поправки.

\section{7. ПРИМЕНЕНИЕ МЕТОДА ТУННЕЛЬНОГО ВОЗМУЩЕНИЯ}

Как показано в разделе 6 , изменение потенциала в классически запрещенной области приводит к экспоненциально малому возмущению спектра. Данные поправки представляют интерес, если в задаче присутствует экспоненциальное квазивырождение спектра, поскольку тогда малое возмущение энергий может привести к существенному изменению собственных функций, а следовательно, и динамики системы. Простейшей подобной системой является двухъямный потенциал. Учитывая результаты раздела 2, исследование двухъямного потенциала можно свести к исследованию пары одноямных потенциалов, для которых применима теорема 2.

Для начала рассмотрим влияние деформации потенциального барьера на туннелирование. Пользуясь полученными результатами, построим контрпример к работе [13]. Затем приведем независимое доказательство корректности условия 3 теоремы 1, опирающееся на оценки из следствия 2. 
Рассмотрим влияние деформации потенциального барьера на резонансное туннелирование в двойной потенциальной яме. Пусть двухъямный потенциал $V(x)$ удовлетворяет требованиям теоремы $1, E_{1,2}$ - пара квазивырожденных собственных значений оператора $\widehat{H}$ и соответствующие собственные функции билокализованны. Добавим к потенциалу $V$ функцию $f(x)$ такую, что носитель $f(x)$ лежит между центром потенциального барьера и точкой поворота. Для определенности можно считать, что возмущение имеет место на правой стороне барьера.

Тогда, используя результаты теоремы 1 и 2, легко доказать следующую теорему.

Теорема 3. При гладкой деформации одной, для определенности правой, стороны потенциального барьера разрушается двойная локализация собственных функиий, и для величины расщепления справедлива формула

$$
\Delta=\left\langle f(x) \psi_{\mathrm{r}}, \psi_{\mathrm{r}}\right\rangle(1+o(1)) .
$$

Аналогичная задача в случае симметричного потенциала рассматривалась в работе [18], где был получен только показатель экспоненты, такой же, как в оценке из следствия 2.

Теперь покажем с помощью формулы для туннельного возмущения, что результаты, приведенные в работе [13], неверны.

В работе [13] рассматривается двухъямный несимметричный потенциал для энергий, близких к положениям равновесия потенциала. Предполагается, что потенциал является в точности квадратичным в некоторой окрестности областей классического движения, т. е. в некоторых конечных окрестностях минимумов потенциала. Для вычисления величины расщепления применялся метод двухуровневой аппроксимации. В качестве потенциалов левой и правой потенциальных ям выбирались осцилляторы с соответствующими частотами.

Ошибка состоит в том, что при таком выборе потенциалов левой и правой потенциальных ям полученный результат для величины расщепления оказывается меньше величины погрешности данного метода. Из такого подхода следует, что локализация собственных функций и наличие туннельной транспортации не зависят от выбора гладкого участка потенциального барьера, соединяющего те области, где потенциал квадратичен. Такие вычисления приводят к неверному результату, поскольку деформация потенциального барьера вне областей квадратичности потенциала приведет к разрушению туннелирования (теорема 3). В самом деле, величина расщепления будет экспоненциально больше, чем полученная в работе [13]. В качестве контрпримера можно рассмотреть любой симметричный двухъямный потенциал, удовлетворяющий всем условиям работы [13], к которому добавлено несимметричное возмущение потенциального барьера.

Далее приведем независимое доказательство корректности условий теоремы 1 , используя формулы туннельного возмущения спектра. Условие 3 теоремы 1 сформулировано в терминах спектров операторов $\widehat{H}_{\ell, \mathrm{r}}$ с одноямными потенциалами $V_{\ell, \mathrm{r}}(x)$. Выбор потенциалов $V_{\ell, \mathrm{r}}$ не является однозначным, как видно из условий, наложенных на потенциалы $V_{\ell, \mathrm{r}}$. Пусть выбраны другие потенциалы $W_{\ell, \mathrm{r}}$. Тогда они отличаются от исходных потенциалов $V_{\ell, \mathrm{r}}$ только в классически запрещенной области. Как следует из теоремы 1 условие существования числа $\lambda$ такого, что $\left|E_{\mathrm{r}}-E_{\ell}\right|=$ $\delta(\hbar)(\lambda+O(\hbar))$, не зависит от свободы в выборе $V_{\ell, \mathrm{r}}$ и характеризует двухъямный 
потенциал $V$. Докажем этот факт непосредственно, используя формулу для туннельного возмущения.

Пусть

$$
\widehat{K}_{i}=-\hbar^{2} \frac{d^{2}}{d x^{2}}+W_{i}(x), \quad i=\ell, \mathrm{r} .
$$

Предположим, что для собственных значений $E_{\ell, \mathrm{r}}$ операторов $\widehat{H}_{\ell, \mathrm{r}}$ справедливо условие 3 теоремы 1 , т. е. существует $\lambda$ такое, что

$$
\left|E_{\mathrm{r}}-E_{\ell}\right|=\delta(\hbar)(\lambda+O(\hbar)) .
$$

Пусть $\psi_{\ell, \mathrm{r}}$ - собственные функции, соответствующие собственным значениям $E_{\ell, \mathrm{r}}$. Нужно доказать, что у операторов $\widehat{K}_{\ell, \mathrm{r}}$ существуют собственные значения $k_{\ell, \mathrm{r}}$, близкие к $E_{\ell, \mathrm{r}}$, и такие, что

$$
\left|k_{\mathrm{r}}-k_{\ell}\right|=\delta(\hbar)(\lambda+O(\hbar))
$$

Введем обозначение

$$
f_{i}(x)=W_{i}(x)-V_{i}(x), \quad i=\ell, \mathrm{r} .
$$

Тогда из условий, наложенных на потенциалы $V_{i}$ и $W_{i}$, следует, что

$$
f_{\ell}(x) \equiv 0 \quad \text { при } \quad x \leqslant b, \quad f_{\mathrm{r}}(x) \equiv 0 \quad \text { при } \quad x \geqslant a .
$$

Положим

$$
S_{\ell}=\int_{x_{\ell}}^{b}|p(x)| d x, \quad S_{\mathrm{r}}=\int_{a}^{x_{\mathrm{r}}}|p(x)| d x .
$$

Применяя оценку из следствия 2 , получаем

$$
k_{i}-E_{i}=\exp \left(-\frac{2 S_{i}+o(1)}{\hbar}\right) .
$$

Поскольку точки $a$ и $b$ выбраны так, что $a<c<b$, а точка $c$ является центром потенциального барьера с точки зрения действия,

$$
\int_{x_{\ell}}^{c}|p| d x=\int_{c}^{x_{\mathrm{r}}}|p| d x
$$

получаем, что величина $\left|k_{i}-E_{i}\right|$ экспоненциально меньше, чем $\delta(\hbar)$. Следовательно,

$$
\frac{\left|k_{\mathrm{r}}-k_{\ell}\right|}{\delta}=\frac{\left|E_{\mathrm{r}}-E_{\ell}\right|}{\delta}+O(\hbar)=\lambda+O(\hbar),
$$

что и требовалось доказать.

ПРИЛОЖЕНИЕ

\section{Некоторые теоремы из теории линейных операторов}

Широко известны следующие утверждения.

Лемма 1 (норма резольвенты). Пусть Г - гилъбертово пространство, оператор А самосопряжен. Тогда, если $\lambda \notin \operatorname{Spectre}(A)$, mо

$$
\left\|(A-\lambda)^{-1}\right\|=\frac{1}{d(\lambda, \operatorname{Spectre}(A))},
$$

где $d$ - расстояние на прямой. 
Лемма 2. Пусть Г - гилъбертово пространство, оператор А самосопряжен. Пусть задана квазимода и:

$$
\|u\|=1, \quad\|(A-\lambda) u\| \leqslant \varepsilon .
$$

Тогда

$$
d(\lambda, \operatorname{Spectre}(A)) \leqslant \varepsilon .
$$

ТЕОРема 4 (равенство числа мод и квазимод). Пусть для самосопряженного оператора $A$ в гильбертовом пространстве Г имеются $n$ ортонормированных квазимод:

$$
\left\langle u_{i}, u_{j}\right\rangle=\delta_{i j}, \quad\left\|(A-\lambda) u_{i}\right\| \leqslant \varepsilon, \quad i, j=1,2, \ldots, n .
$$

Тогда существуют как минимум $n$ ортогональных собственных функций $\psi_{k}$ оператора А с собственными значениями из в-окрестности $\lambda$ :

$$
\left(A-\lambda_{k}\right) \psi_{k}=0, \quad\left|\lambda-\lambda_{k}\right| \leqslant \varepsilon, \quad k=1,2, \ldots, n .
$$

Теорема 5 (разложение квазимоды). Пусть Г - гильбертово пространство, оператор А самосопряжен. Пусть задана квазимода $u \in \Gamma,\|u\|=1,\|(A-\lambda) u\| \leqslant \varepsilon$. Предположим, что на интервале $(\lambda-\varepsilon, \lambda+\varepsilon)$ содержится всего одна точка спекmpa оператора $A$ :

$$
A \psi=\mu \psi, \quad|\lambda-\mu|<\varepsilon .
$$

Тогда для квазимоды и имеет место разложение

$$
u=\langle u, \psi\rangle \psi+\widetilde{u}, \quad\|\tilde{u}\| \leqslant \frac{\varepsilon}{d},
$$

где $d$ - расстояние от $\lambda$ до спектра А без учета точки $\mu$.

ЗАмечАние 1. Теорему 5 имеет смысл применять в том случае, когда известно, что $d \gg \varepsilon$. Эта теорема может дать представление о виде точной собственной функции.

Следующая теорема практически идентична теореме, доказанной в работе [9], несколько изменены формулировка и доказательство.

ТЕОРЕма 6. Пусть в гилъбертовом пространстве Г задан самосопряженный оператор А. Пусть выполнены следующие условия.

1. Для оператора А задан набор из $n$ ортонормированных квазимод:

$$
\left\langle u_{i}, u_{j}\right\rangle=\delta_{i j}, \quad\left\|(A-\lambda) u_{i}\right\| \leqslant \varepsilon, \quad i, j=1,2, \ldots, n .
$$

2. Пусть d-расстояние от $\lambda$ до спектра А при условии, что п ближайших точек не учитываются (число точек считается с учетом кратности). Справедлива оченка

$$
d>\sqrt{2 n} \varepsilon .
$$

3. Найдены собственные значения $\mu_{i}$ и соответствующие собственные векторы $z_{i}, i=\overline{1, n}$, матрицы, состоящей из элементов

$$
V_{i j}=\left\langle(A-\lambda) u_{i}, u_{j}\right\rangle, \quad i, j=1,2, \ldots, n .
$$

Собственные векторы $z_{i}$ выбраны ортонормированными. 
Тогда существуют $y_{i} \in \Gamma$ такие, что

$$
\begin{gathered}
y_{i}=v_{i}+w_{i}, \quad i=1,2, \ldots, n, \\
v_{i}=\sum_{j=1}^{n} z_{i}^{j} u_{j}, \quad\left\|w_{i}\right\| \leqslant \frac{\sqrt{2 n} \varepsilon}{d-\sqrt{2 n} \varepsilon}, \\
\left\|\left(A-\lambda-\mu_{i}\right) y_{i}\right\| \leqslant \frac{4 n \varepsilon^{2}}{d-\sqrt{2 n} \varepsilon} .
\end{gathered}
$$

ЗАмЕчАниЕ 2. Если $d \gg \varepsilon$, то условие 2 выполняется автоматически, и вычисление собственных значений матрицы $V$ позволяет получить спектр оператора $A$ с точностью $O\left(\varepsilon^{2} / d\right)$.

ДоКАЗАТЕЛЬСТво тЕОРЕмЫ 6. Из первого условия данной теоремы следует, что на интервале существуют не менее $n$ точек спектра оператора $A$, а из второго условия данной теоремы следует, что их не более $n$. Обозначим через $\psi_{i}$ собственные функции оператора $A$, отвечающие собственным значениям $\lambda_{i}$ из рассмотренного интервала. Тогда $d$ - это расстояние от точки $\lambda$ до спектра $A$ без учета точек $\lambda_{i}, i=1, \ldots, n$.

Пусть $E$ - ортогональный проектор на линейную оболочку векторов $u_{i}, i=$ $1, \ldots, n$, а $E^{\prime}=I-E$. Определим операторы

$$
V=(A-\lambda) E+E(A-\lambda)-E(A-\lambda) E, \quad A_{0}=A-V .
$$

Покажем, что $V$ - конечномерный оператор. Пусть $u_{1}, \ldots, u_{n}, \ldots, u_{\mathrm{r}}$ - ортонормированный базис в пространстве, образованном набором векторов $u_{1}, \ldots, u_{n}$, $(A-\lambda) u_{1}, \ldots,(A-\lambda) u_{n}$. Пусть функция $\phi \in \Gamma$ ортогональна всем $u_{i}, i=\overline{1, r}$. Тогда

$$
\|E(A-\lambda) \phi\|^{2}=\langle E(A-\lambda) \phi, E(A-\lambda) \phi\rangle=\langle(A-\lambda) E(A-\lambda) \phi, \phi\rangle=0,
$$

поскольку функция $\phi$ ортогональна образу оператора $(A-\lambda) E$. Следовательно, размерность оператора $E(A-\lambda)$ не превосходит $r \leqslant 2 n$. Остальные слагаемые в определении $V$, очевидно, конечномерны.

Докажем, что $\|V\| \leqslant \sqrt{2 n \varepsilon}$. Очевидно, что

$$
\|(A-\lambda) E\| \leqslant \sqrt{n} \varepsilon .
$$

Оценим норму оператора $E(A-\lambda)$. Поскольку $E(A-\lambda)$ сопряжен к $(A-\lambda) E$, имеем

$$
\|E(A-\lambda)\| \leqslant \sqrt{n} \varepsilon
$$

Пусть $\phi \in \Gamma$ и $\|\phi\|=1$. Тогда

$$
\|V \phi\|^{2}=\left\|E^{\prime}(A-\lambda) E \phi+E(A-\lambda) \phi\right\|^{2} \leqslant 2 n \varepsilon^{2}+2\left\langle E^{\prime}(A-\lambda) E \phi, E(A-\lambda) \phi\right\rangle=2 n \varepsilon^{2} .
$$


Покажем, что для оператора $A_{0}$ значение $\lambda$ есть изолированная точка спектра кратности $n$. Из определения $A_{0}$ и того, что $E u_{i}=u_{i}, i=\overline{1, n}$, вытекает, что

$$
\left(A_{0}-\lambda\right) u_{i}=(A-\lambda) u_{i}-V u_{i}=0, \quad i=1,2, \ldots, n .
$$

Поскольку $\|V\| \leqslant \sqrt{2 n} \varepsilon$, любая собственная функция оператора $A$ является квазимодой оператора $A_{0}$ и наоборот. Поэтому на интервале $(\lambda-d+\sqrt{2 n} \varepsilon, \lambda+d-\sqrt{2 n} \varepsilon)$ оператор $A_{0}$ имеет ровно $n$ точек спектра с учетом кратности. В противном случае возникает противоречие с условием 2 данной теоремы. Из леммы 1 получаем

$$
\left\|\left(A_{0}-\lambda\right)^{-1} E^{\prime}\right\| \leqslant(d-\sqrt{2 n} \varepsilon)^{-1} .
$$

Таким образом, мы имеем представление оператора $A$ в виде суммы оператора $A_{0}$ и малого конечномерного оператора $V$. Спектр и собственные векторы оператора $A_{0}$ в окрестности точки $\lambda$ известны. Для получения поправок к собственным векторам и числам оператора $A$ можно применить теорию возмущений в случае изолированного вырожденного собственного значения $\lambda$.

Пусть $v_{i}$ такие, что $E v_{i}=v_{i}$, являются собственными функциями оператора $E V E$. Найдутся $n$ таких функций $v_{i}$, так как оператор $E V E$ конечномерен и имеет размерность $n$ :

$$
E V v_{i}=\mu_{i} v_{i}, \quad\left\|v_{i}\right\|=1, \quad i=1,2, \ldots, n .
$$

Пусть $w_{i}=-\left(A_{0}-\lambda\right)^{-1} E^{\prime} V v_{i}$. Из определений $v_{i}$ и $w_{i}$ следует, что

$$
\begin{gathered}
A_{0} v_{i}=A_{0} E v_{i}=\lambda v_{i}, \\
\left(V-\mu_{i}\right) v_{i}=\left(E^{\prime}+E\right)\left(V-\mu_{i}\right) v_{i}=E^{\prime} V v_{i}, \quad\left(A_{0}-\lambda\right) w_{i}=-E^{\prime} V v_{i} \\
\left|\mu_{i}\right| \leqslant\|V\| \leqslant \sqrt{2 n \varepsilon}, \quad\left\|V-\mu_{i}\right\| \leqslant\|V\|+\left|\mu_{i}\right| \leqslant 2 \sqrt{2 n \varepsilon} \\
\left\|w_{i}\right\|=\left\|\left(A_{0}-\lambda\right)^{-1} E^{\prime} V v_{i}\right\| \leqslant\left\|\left(A_{0}-\lambda\right)^{-1} E^{\prime}\right\|\|V\|\left\|v_{i}\right\| \leqslant \frac{\sqrt{2 n} \varepsilon}{d-\sqrt{2 n} \varepsilon} \\
\left\|\left(A-\lambda-\mu_{i}\right)\left(v_{i}+w_{i}\right)\right\|=\left\|\left(A_{0}-\lambda+V-\mu_{i}\right)\left(v_{i}+w_{i}\right)\right\|= \\
=\left\|\left(V-\mu_{i}\right) w_{i}\right\| \leqslant\left\|\left(V-\mu_{i}\right)\right\|\left\|w_{i}\right\| \leqslant \frac{4 n \varepsilon^{2}}{d-\sqrt{2 n} \varepsilon} .
\end{gathered}
$$

Таким образом, мы получили, что $v_{i}+w_{i}$ являются квазимодами с собственными значениями $\lambda+\mu_{i}$, где $i=1,2, \ldots, n$.

Искать $v_{i}$ и $\mu_{i}$ можно, используя матричные элементы оператора $V$ в базисе, содержащем векторы $u_{i}, i=1,2, \ldots, n$. Тогда

$$
V_{i, j}=\left\langle V u_{i}, u_{j}\right\rangle=\left\langle(A-\lambda) E u_{i}, u_{j}\right\rangle=\left\langle(A-\lambda) u_{i}, u_{j}\right\rangle
$$

Собственные векторы этой матрицы суть коэффициенты разложения вектора $v_{i}$ по векторам $u_{j}$, а собственными значениями являются именно $\mu_{i}, i=1,2, \ldots, n$. Теорема доказана.

Благодарности. Автор благодарен М. В. Карасеву за постановку задачи, плодотворные обсуждения и помощь в подготовке статьи. Работа выполнена на кафедре прикладной математики МИЭМ в рамках Программы фундаментальных исследований НИУ ВШЭ. 


\section{Список литературы}

[1] M. Razavy, Quantum Theory of Tunneling, World Sci., Singapore, 2003.

[2] J. Ankerhold, Quantum Tunneling in Complex Systems. The Semiclassical Approach, Springer Tracts in Modern Physics, 224, Springer, Berlin, 2007.

[3] F. Hund, Z. Phys., 40:10 (1927), 742-764.

[4] Л. Д. Ландау, Е. М. Лифшиц, Теоретическая физика, т. 3: Квантовая механика. Нерелятивистская теория, Наука, М., 1974.

[5] С. Ю. Доброхотов, В. Н. Колокольцов, В. П. Маслов, ТМФ, 87:3 (1991), 323-375.

[6] A. Gangopadhyay, M. Dzero, V. Galitski, Phys. Rev. B, 82:2 (2010), 024303, 13 pp., arXiv: 1005.0652.

[7] С. Ю. Славянов, В. Лай, Специальные функиии. Единая теория, основанная на анализе особенностей, Невский Диалект, СПб, 2002.

[8] M. M. Nieto, V.P. Gutschick, C. M. Bender, F. Cooper, D. Strottman, Phys. Lett. B, 163:5-6 (1985), 336-342.

[9] Т. Ф. Панкратова, "Квазимоды и экспоненциальное расщепление собственных значений", Проблемы математической физики, т. 11: Дифференииалъные уравнения и теория рассеяния, Изд-во Ленинградского ун-та, Л., 1986, 167-177.

[10] B. Helffer, J. Sjóstrand, Commun. Partial Differential Equations, 9:4 (1984), 337-408.

[11] B. Helffer, J. Sjóstrand, Ann. Inst. H. Poincaré, 42:2 (1985), 127-212.

[12] Т. Ф. Панкратова, Зап. научн. сем. ЛОМИ, 195 (1991), 103-112.

[13] D.-Y. Song, Ann. Phys., 323:12 (2008), 2991-2999, arXiv: 0803.3113.

[14] S. Agmon, Lectures on Exponential Decay of Solutions of Second-Order Elliptic Equations: Bounds on Eigenfunctions of N-Body Schrödinger Operators, Mathematical Notes, 29, Princeton Univ. Press, Princeton, NJ, 1982.

[15] B. Simon, J. Funct. Anal., 63:1 (1985), 123-136.

[16] B. Simon, Bull. AMS, 8:2 (1983), 323-326.

[17] С. Ю. Доброхотов, В. Н. Колокольцов, ТМФ, 94:3 (1993), 426-434.

[18] G. Jona-Lasinio, F. Martinelli, E. Scoppola, Commun. Math. Phys., 80:2 (1981), 223-254.

[19] М. В. Федорюк, Асимптотические методы для линейных обыкновенных дифберенциалъных уравнений, Наука, М., 1977.

Поступила в редакцию 17.03.2013, после доработки 15.07.2013 